\title{
Hyphema from Iris Melanocytoma
}

\author{
Neel D. Pasricha ${ }^{\mathrm{a}}$ Michael I. Seider ${ }^{\mathrm{a}-\mathrm{c}}$ \\ ${ }^{a}$ Department of Ophthalmology, University of California, San Francisco, CA, USA; ${ }^{b}$ Department of \\ Ophthalmology, The Permanente Medical Group, San Francisco, CA, USA; ' Department of Ophthalmology, \\ California Pacific Medical Center, San Francisco, CA, USA
}

\section{Established Facts}

- Iris melanocytoma presents at a young age, may grow slowly, and has a characteristic jet-black color and corrugated surface.

\section{Novel Insights}

- Iris melanocytoma may present with spontaneous, recurrent hyphema.

\section{Keywords}

Iris melanocytoma · Hyphema · Ocular oncology

\section{Abstract}

Using multimodal imaging, we demonstrate a classic iris melanocytoma presenting with recurrent, spontaneous hyphema in a young female patient. The patient has been observed without tumor growth. Hyphema has not been previously reported to occur with iris melanocytoma.

(c) 2018 S. Karger AG, Basel

\section{Introduction}

Iris melanocytoma typically presents at a young age, usually demonstrates minimal growth over many years, is classically jet-black in color, often has cystic changes with a corrugated surface, and may produce satellite le- sions $[1,2]$. Although spontaneous hyphema has been reported with iris melanoma, it has not been reported with iris melanocytoma [1-4].

\section{Case Report}

A 13-year-old girl presented with a left iris lesion characteristic of melanocytoma which, according to her mother, had exhibited minimal growth since detected at the age of 3 years. The patient complained of a 4-month history of recurrent hyphema in this eye associated with minimal trauma such as eye rubbing, exercise, or laughing and which spontaneously resolved over 1-3 days with activity restriction. On examination at presentation, a small hyphema adjacent to an inferonasal darkly pigmented iris mass with an irregular surface with cystic changes was noted along with satellite lesions, corectopia, and an appearance otherwise consistent with iris melanocytoma (Fig. 1a, b). A temporal, superficial intratumoral vessel was also identified.

Ultrasound biomicroscopy images of her iris mass demonstrated a highly reflective nodular mass appearing to originate from the mid and anterior iris with an irregular surface and sharp, well-
KARGER

() 2018 S. Karger AG, Basel

E-Mail karger@karger.com

www.karger.com/oop
Michael. I. Seider

The Permanente Medical Group 1635 Divisadero Street

San Francisco, CA 94115 (USA)

E-Mail Michael.I.Seider@kp.org 

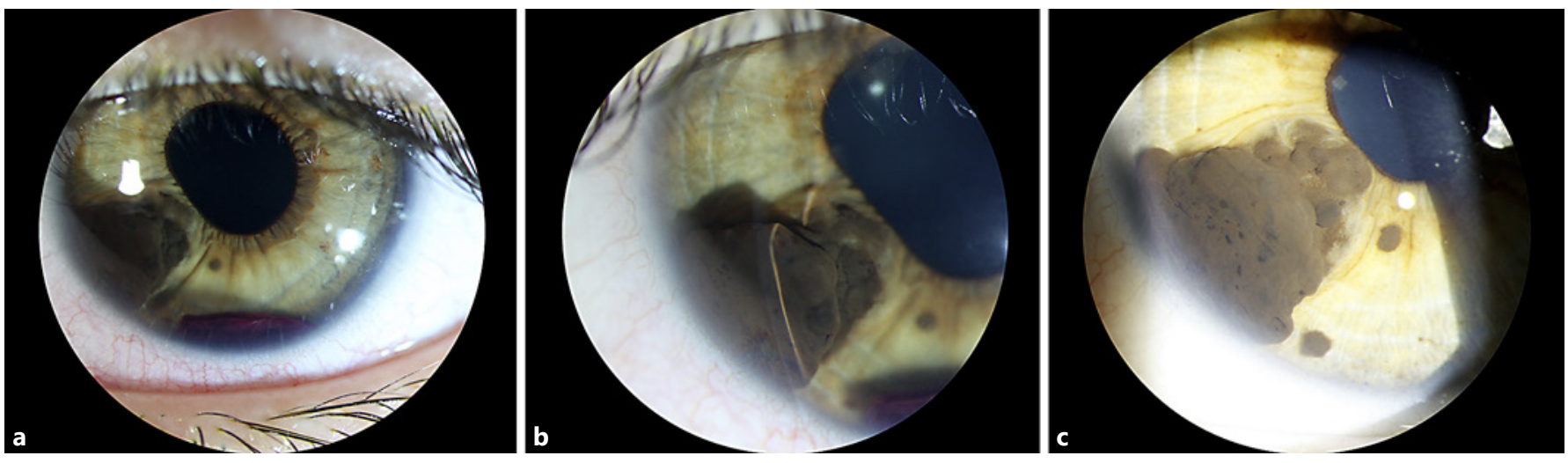

Fig. 1. Slit lamp photos. a Diffuse illumination showing a large, darkly pigmented mass of the left iris from 7:00 to 8:30 o'clock causing distortion of the pupillary margin as well as a small associated hyphema. One satellite lesion is seen on the anterior iris surface at 7 o'clock. b High magnification slit beam cross-section- al view of the iris mass highlights its irregular surface. c Diffuse illumination 17 months after initial presentation demonstrating a stable large, darkly pigmented iris mass with subtle changes in the anterior surface corrugations. No hyphema was present at this visit.

Fig. 2. Ultrasound biomicroscopy. a Transverse (top) and longitudinal (bottom) 8 o'clock views demonstrating an anterior iris mass without ciliary body or posterior iris involvement showing "collar-stud"type configuration. $\mathbf{b}$ Transverse (top) and longitudinal (bottom) 8 o'clock views of the same anterior iris mass 17 months later demonstrating stable configuration without growth.
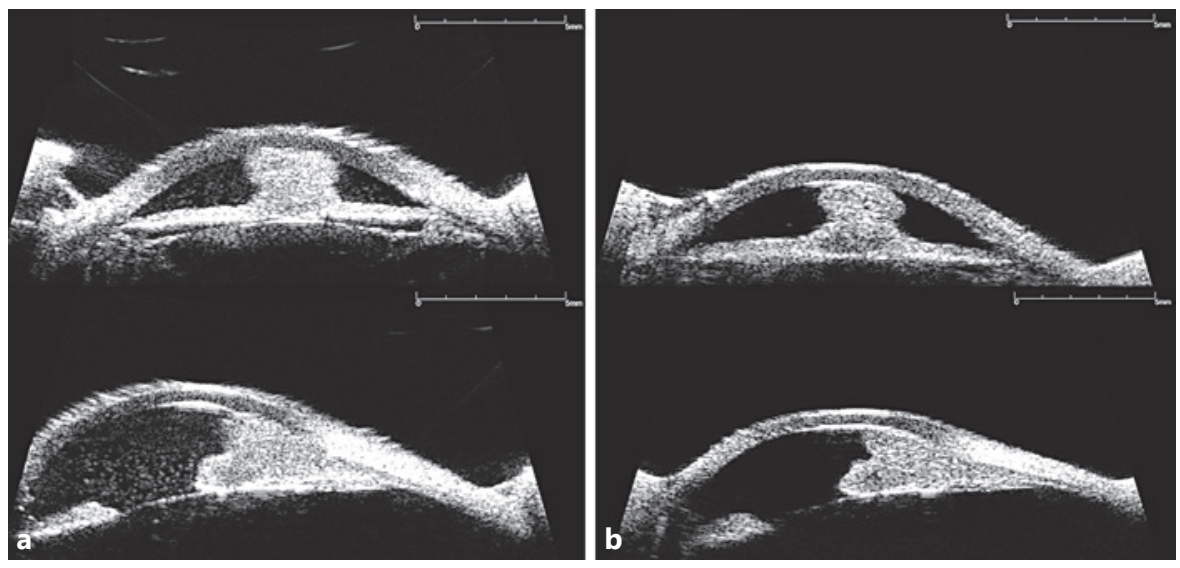

defined edges characteristic of iris melanocytoma (Fig. 2) [5]. The mass did not involve the posterior iris or ciliary body.

The patient was observed without intervention or activity restriction. After 17 months of follow-up, the lesion has remained stable on examination and ultrasound although the previously visible tumoral vessel appears to have regressed (Fig. 1c). The patient continues to experience recurrent layering hyphema approximately once every 2 months but fortunately no corneal or lenticular pigmentation, intraocular pressure elevation, or optic neuropathy has occurred. We continue to manage conservatively without intervention.

\section{Discussion}

We describe an iris lesion exhibiting all the classic features of iris melanocytoma that has caused recurrent hyphema. To our knowledge, hyphema has not been previ- ously reported to occur with iris melanocytoma, but has been reported to occur with iris melanoma. Fortunately, our patient's lesion is not consistent with iris melanoma in that it has been present since childhood, shows no growth over our observation interval, and has no evidence of invasion into the surrounding iris stroma or ciliary body. Although recurrent, the iris melanocytomaassociated hyphemas in this case have not been associated with secondary ocular damage.

The reason for spontaneous hyphema in this case is unclear. Previous studies have noted that uveal nevi and melanomas can grow and/or become more pigmented in association with puberty, and this may underlie the new change in this lesion [6]. In addition, spontaneous necrosis of iris melanocytoma has been reported on numerous occasions [7]. Tumor necrosis may be implicated in the 
cause of spontaneous hyphema in this case, especially because of cystic spaces seen in the tumor on ultrasound as well as the mild change in the surface contour of the tumor over time.

In summary, we describe a case of iris melanocytomaassociated hyphema. This rare co-occurrence did not result in ocular damage or indicate imminent tumor growth and has been safely observed.

\section{Statement of Ethics}

Subjects gave their informed consent and the study protocol was deemed exempt from review by the Committee on Human Research.

\section{Disclosure Statement}

The authors have no sponsorship or funding arrangements relating to their research and no possible conflicts of interest.

\section{Funding Sources}

None.

\section{References}

Hyphema from Iris Melanocytoma
1 Shields CL, Shields PW, Manalac J, Jumroendararasame C, Shields JA: Review of cystic and solid tumors of the iris. Oman J Ophthalmol 2013;6:159-164.

2 Demirci H, Mashayekhi A, Shields C, et al: Iris melanocytoma: clinical features and natural course in 47 cases. Am J Ophthalmol 2005; 139:468-475.

3 Song WK, Yang WI, Lee SC: Iris naevus with recurrent spontaneous hyphema simulating an iris melanoma. Eye (Lond) 2009;23:14861488 .
4 Territo C, Shields CL, JShields JA, et al: Natural course of melanocytic tumors of the iris. Ophthalmology 1988;95:1251-1255.

5 Malandrini A, Mittica V, Tosi GM, et al: Clinical and ultrasound biomicroscopic features in iris melanocytoma. Ophthalmic Surg Lasers Imaging 2009;40:46-49.

6 Shields JA, Shields CL: Intraocular Tumors: An Atlas and Textbook. Philadelphia, Lippincott Williams \& Wilkins, 2008, pt 1, p 160.

7 JShields JA, Annesley WH Jr, Spaeth GL: Necrotic melanocytoma of iris with secondary glaucoma. Am J Ophthalmol 1977;84:826829. 